\title{
Primer Congreso Internacional de Desarrollo Regional: Diálogos para construir región - CINDER
}

\section{First International Congress on Regional Development: Dialogues to build a region - CINDER}

\section{Lady Johanna Peñaloza Farfán ${ }^{1}$ \\ Brenda González Cuellar}

O........

Recepción: 15/12/2020
.

Aprobación: 28/10/2021
O

Publicación: 22/12/2021

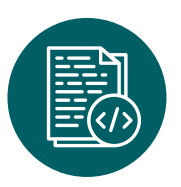

Para citar este artículo

Peñaloza Farfán, L. J., \& González Cuellar, B. (2021). Primer Congreso Internacional de Desarrollo Regional: Diálogos para construir regiónCINDER. Indagare, (9), 96-102.

https://doi.org/10.35707/indagare/910

\footnotetext{
1 Grupo de investigación EULOGOS. Universidad de Ibagué. ORCID: 0000-0003-3920-7634. Correo electrónico: lady.penaloza@unibague.edu.co

2 Grupo de investigación EULOGos. Universidad de Ibagué. ORCID: 0000-0002-2216-7634.

Correo electrónico: brenda.gonzalez@unibague.edu.co
} 


\section{Resumen}

El Primer Congreso Internacional de Desarrollo Regional, Diálogos para construir región, se creó con la necesidad de establecer espacios de diálogo que visibilicen proyectos regionales a partir de cuatro líneas de investigación: Políticas regionales y regiones, Globalización y regiones, Equidad, paz, participación y regionalización y Conocimiento y región. Así pues, se llevó a cabo los días 22 y 23 de octubre de 2020, contando con la participación de treinta y ocho ponencias de investigadores nacionales e internacionales, que por medio de sus aportes permitieron evidenciar dinámicas de participación, contribución y transformación para el desarrollo regional.

\section{Abstract}

The First International Congress on Regional Development, Dialogues to build a region, was created with the need to establish spaces for dialogue that make regional projects visible based on four lines of research: Regional policies and regions, Globalization and regions, Equity, peace, participation and regionalization, and Knowledge and region. Thus, it was held on October $22^{\text {nd }}$ and $23^{\text {rd }}, 2020$, with the participation of thirty-eight speeches by national and international researchers, who through their contributions made it possible to demonstrate dynamics of participation, contribution and transformation towards regional development.

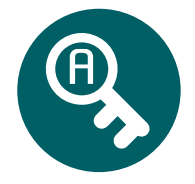

Palabras claves: Región, desarrollo, políticas, globalización, equidad, paz, participación, regionalización.

Key words: Region, development, policies, globalization, equity, peace, participation, regionalization.

\section{Introducción}

Al evidenciar la necesidad de generar escenarios de dialogo que promuevan conversaciones sobre la diversidad regional de Colombia y el subcontinente, el Primer Congreso Internacional de Desarrollo Regional: Diálogos para construir región (CINDER), se fundamenta en la necesidad de visibilizar proyectos regionales y, del mismo modo, comprender el contexto actual, que evidentemente influye en el desarrollo de estos. Teniendo en cuenta lo anterior, se destaca la pertinencia de crear espacios que permitan suscitar diálogos de intercambio social y académicos sobre componentes que podrían originar nuevas oportunidades a través la reflexión (Universidad de Ibagué, 2020). De acuerdo con Rueda (2014), las investigaciones regionales tienen perspectivas 
en común y deben ser interpretados desde un punto de vista interdisciplinar que permita analizar a profundidad otros ámbitos, como por ejemplo el geográfico-cultural.

De este modo, CINDER establece la importancia de aportar al fortalecimiento de iniciativas locales y movimientos sociales, al igual que la participación en espacios de estructuración, financiación y ejecución de estrategias que favorecen a las regiones. Con base en lo anterior, el congreso consideró fundamental contar con la participación de investigadores, académicos, docentes, estudiantes, activistas, líderes y demás actores regionales que, sin duda, representan un rol fundamental en este espacio académico diseñado para compartir perspectivas regionales (Universidad de Ibagué, 2020). Siguiendo a Azuero et al., (2015), en la actualidad es pertinente que los actores regionales trabajen en articulación, partiendo de experiencias que aporten al desarrollo local, puesto que existen múltiples necesidades del territorio que no se pueden resolver de manera individual y que, al ser trabajadas en conjunto, representan una valiosa oportunidad para estructurar proyectos desde diferentes perspectivas y habilidades interdisciplinares. Para hacer visible este propósito se realizó un logo que fue usado en todos los espacios y piezas comunicativas, ver la Figura 1.

El CINDER se desarrolla en el marco de cuatro líneas de investigación relacionadas con políticas que pueden aportar o suponer un obstáculo para el desarrollo regional, la problematización del lugar que ocupan las regiones en dinámicas globales desde el punto de vista legal e ilegal, el papel de los actores locales o regionales en la construcción del futuro y en el planteamiento de dinámicas conflictivas que los afectan desde una perspectiva relacionada con equidad, paz, participación y regionalización y, por último, el modo en que la construcción y circulación del conocimiento se produce regionalmente aportando en las transformaciones del territorio (Universidad de Ibagué, 2020).

Figura 1. Congreso Internacional de Desarrollo Regional: Diálogos para construir región

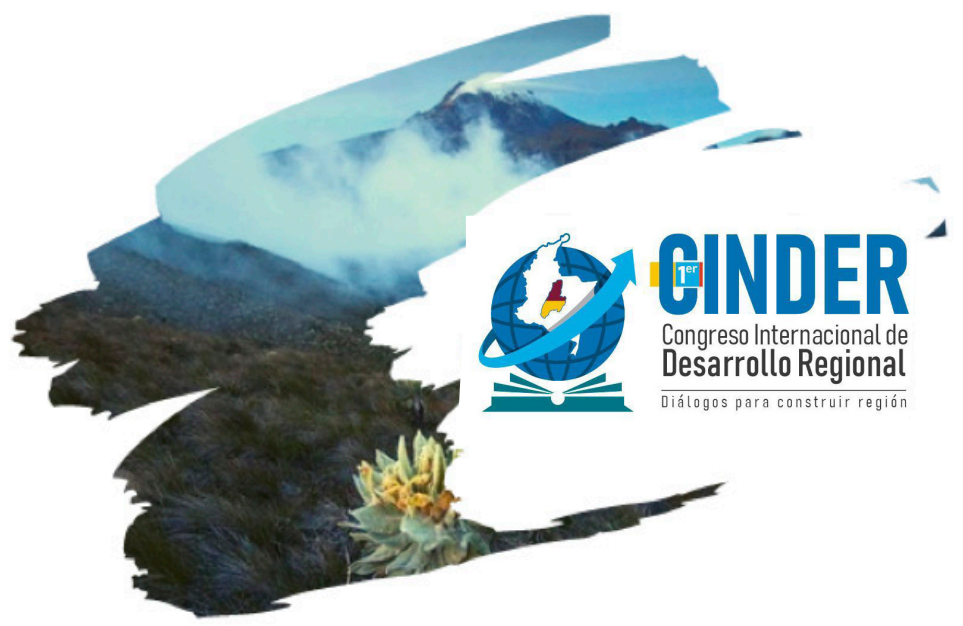

Fuente: Comité organizador CINDER 


\section{Desarrollo}

El Primer Congreso Internacional de Desarrollo Regional (CINDER) es una iniciativa que nace del interés por crear espacios de diálogo que permitan debatir sobre la diversidad regional de Colombia, así como del subcontinente. De este modo, surge el interés por identificar y visibilizar proyectos estructurados bajo la premisa del desarrollo de las regiones y los territorios, y comprender en qué medida y de qué forma influye la actual coyuntura global en el desarrollo de estas iniciativas de investigación (Universidad de Ibagué, 2020).

Teniendo en cuenta lo anterior, es interés del congreso impulsar la participación y el conocimiento de perspectivas regionales, proponer escenarios de diálogo y reflexión sobre las características y elementos que los componen, así como incentivar al desarrollo de debates de saber sobre los componentes potenciales para la creación de nuevas oportunidades articuladas para el fortalecimiento de iniciativas locales y movimientos sociales que participen de espacios de estructuración, financiación y ejecución de políticas que impactan en las regiones (Universidad de Ibagué, 2020).

En este sentido, y de acuerdo con Villaveces-Niño y Sánchez (2015), en el mundo actual el aporte de los movimientos sociales es decisivo para poder generar desarrollos que develen las verdaderas necesidades de las personas, pues como establece Escobar (2002), la innovación y las transformaciones son de quienes conocen y de quienes poseen los conocimientos que se perciben como importantes (citado en Villaveces-Niño \& Sánchez, 2015).

Por otra parte, y con relación al desarrollo del evento, el CINDER fue organizado por la Universidad de Ibagué en articulación con la Universidad del Tolima, el Servicio Nacional de Aprendizaje (SENA), el Centro de Estudios Regionales (CERE) y el Consejo territorial de planeación Tolima; además contó con la importante participación de cuatro ponentes internacionales: Iokiñe Rodríguez de la Universidad de East Anglia localizada en Norwich, Inglaterra; Juan Carlos Ramírez de la Universidad de Los Andes en Bogotá, Colombia; Zoraida Mendiwelso-Bendek de la Universidad de Lincoln en Pensilvania, Estados Unidos; y César Vallejo en representación de la Universidad de Ibagué.

Así pues, el congreso se llevó a cabo los días 22 y 23 de octubre de 2020 de manera virtual, medida que se implementó dada la actual contingencia. En esta oportunidad se contó con la participación de treinta y ocho (38) ponencias provenientes de instituciones nacionales como la Universidad Externado de Colombia, Corporación Universitaria Minuto de Dios, Universidad Industrial de Santander (UIS), Universidad Autónoma de Bucaramanga, Universidad Jorge Tadeo Lozano, Universidad del Rosario, Fundación Universidad América, Universidad Militar Nueva Granada, 
Universidad Pedagógica Nacional, Universidad Cooperativa de Colombia, la Granja SENA Espinal, Pontificia Universidad Javeriana, Instituto Colombiano de Antropología e Historia-ICANH, Universidad San Buenaventura, Centro de Estudios Regionales (CERE Tolima), Universidad Nacional de Colombia, Universidad La Gran Colombia, Universidad Pedagógica Nacional, Universidad San Buenaventura, Escuela Normal Superior de Neiva, Escuela Popular Claretiana, Universidad Autónoma de Occidente, Universidad de La Salle y, por supuesto, la Universidad de Ibagué, Universidad del Tolima y el SENA.

Además, se tuvo la participación de instituciones internacionales como la Universidad de East Anglia, Universidad de Lincoln del Reino Unido, Universidad de Rio de Janeiro, de Brasil, Universidad Politécnica de Valencia, Instituto InGENIO de España, Arizona State University (ASU) de Estados Unidos, Universidad de Valencia, España, Facultad Latinoamericana de Ciencias Sociales, FLACso de México. Todas ellas con aportes importantes en este espacio de diálogos para construir región.

El evento científico tuvo en cuenta cuatro líneas de investigación para su desarrollo, la primera denominada "Políticas regionales y regiones: logros, problemas y retos", que aborda temas relacionados con manejos que refuerzan o impiden el desarrollo regional, y asuntos referentes a la dotación de infraestructura, conflictos emergentes de la explotación minero-energética, gestión de recursos locales/regionales, proceso de planificación territorial y sus implicaciones en las dinámicas regionales y dinámicas relacionadas con las decisiones que afectan a la región (Universidad de Ibagué, 2020).

Teniendo en cuenta lo anteriormente mencionado, es de importancia resaltar la pertinencia de establecer diálogos con relación a la transformación que la región debe asumir como actor de desarrollo con respecto a la generación de nuevos proyectos regionalistas, pero esto depende de la capacidad de sobresalir en el ámbito social y político, así pues, se debe asumir un papel activo, representando proyectos políticos regionales desde la alianza de intereses, potenciando su autoorganización, transformando la comunidad y consiguiendo convertirse en sujeto de su propio desarrollo (Mejía, Molina, Castaña, Londoño \& Álvarez, 2006).

La segunda línea de investigación es titulada "Globalización y regiones: conflictividades, problemas, experiencias exitosas y oportunidades para el desarrollo regional", la cual estudia la forma en que los territorios se vinculan a circuitos políticos, económicos y culturales, teniendo en cuenta el papel de las regiones en contextos globales y analizando diversas perspectivas de los efectos fenomenológicos sobre las maquilas, el turismo y otras dinámicas locales; y, de otro lado, las tácticas que implementan los actores regionales para generar oportunidades y afrontar los efectos negativos de la globalización (Universidad de Ibagué, 2020).

Para tener en cuenta, según Alejandre y Pineda (2011), la tendencia 
globalizadora se establece cuando se generan articulaciones de intercambio diferencial de recursos o productos, además Escobar (2002) manifiesta que cuando se habla de globalización se debe mencionar que, por un lado, este es un fenómeno que desencadena interdependencia entre las regiones del mundo dando paso a la igualdad de condiciones y, por otra parte, es un modelo de desarrollo económico a gran escala que ha sido significativamente influenciado por el neoliberalismo al cual se han vinculado los países deliberadamente (citado en Villaveces-Niño \& Sánchez, 2015).

Otra línea es la designada "Equidad, paz, participación y regionalización: mujeres, niños, jóvenes, grupos étnicos en la construcción regional”, en la que se plantea el rol de los actores locales/regionales en la edificación de sus objetivos futuros y la indagación de las dinámicas conflictivas que se les presentan. El objetivo de esta línea es el de visibilizar las vivencias y redes interregionales que contribuyen al desarrollo de las regiones, además de develar las barreras, tenciones y problemáticas que se presentan sobre la marcha (Universidad de Ibagué, 2020).

En consecuencia, una de las herramientas fundamentales de intervención del desarrollo para validar el sentido adquirido por Región son los referentes a los planes direccionados a la significación de este y al desarrollo regional, aquí es primordial la participación de los actores regionales, teniendo en cuenta las experiencias que posibilitan o restringen el esfuerzo por el progreso del territorio (Mejía, Molina, Castaña, Londoño \& Álvarez, 2006).

Finalmente, la cuarta línea de investigación es “Conocimiento y región: el papel de las universidades en la construcción regional", en la que se discute la forma en que la construcción y divulgación del conocimiento se genera regionalmente, así pues, se pretende plantear cuestionamientos en función de la pertinencia de los saberes y de su utilidad en el progreso territorial, aquí se abordan elementos como la generación y apropiación de tecnologías y la competitividad (Universidad de Ibagué, 2020). Teniendo en cuenta la anterior línea de investigación, es importante mencionar que en la actualidad el conocimiento filosófico y ético emergente de las ciencias humanas y sociales, además del progreso de la ciencia y la tecnología podrán encaminar en una realidad dinámica, transformadora, compleja, incierta y que involucra para los individuos la formación en destrezas y competencia, valores y actitudes que evidencien el pensamiento autónomo, crítico y filosófico (Orozco, 2009 citado en Gallardo, 2015).

\section{Cierre}

El CINDER contó con la participación de académicos e investigadores nacionales e internacionales, también tuvo la participación de estudiantes, docentes y diversos actores regionales, que en conjunto aportaron a este espacio pensado para compartir y dialogar sobre perspectivas regionales. El evento permitió develar la importancia 
de transformaciones institucionales de manera global, en pro de suscitar cambios que permitan el progreso de los territorios, puesto que como lo mencionan Alejandre y Pineda (2011), el mundo evidencia el agotamiento de sus recursos naturales, denotando la importancia de establecer políticas regionales alternativas que aporten a la construcción de nuevas realidades que den paso a la generación de nuevas configuraciones sociopolíticas.

Por consiguiente, se manifiesta la importancia de seguir participando y aportando de espacios de conocimiento que permitan establecer diálogos de saberes que logren evidenciar el esfuerzo de los actores regionales por contribuir al desarrollo regional, que al ser descentralizado y abordado a partir de perspectivas interdisciplinares establecen tejidos regionales que posibilitan la equidad territorial. En este sentido, Elizalde (2003) menciona que el desarrollo local se consigue a través de la producción responsable y el fortalecimiento social con base al desarrollo sostenible (citado por Azuero et al., 2015).

\section{Referencias}

Alejandre, G., \& Pineda, J. (2011). Desarrollo y espacio regional, una aproximación teórica metodológica. Espacios Públicos, 14(30), 192-210. Recuperado de https://www.redalyc.org/articulo.oa?id=676/67618934013

Azuero, A., García, M., \& Betancourt, B. (2015) Aproximación a una experiencia regional de responsabilidad social empresarial en el suroccidente colombiano. Revista de la Facultad de Ciencias Económicas y Administrativas. Universidad de Nariño, 1(1), 120-141. Recuperado de http://www.scielo.org.co/pdf/tend/v17n1/v17n1a07.pdf

Gallardo, C. (2015). Planificación y desarrollo regional. Opción, 31(5), 380-393. Recuperado de https://www.redalyc.org/articulo.oa?id=310/31045570023

Mejía, R., Molina, J., Castaña, S., Londoño, D., \& Álvarez, M. (2006). Significación de región y desarrollo regional desde los planes nacionales de desarrollo 1962-2006. Sophia, (2), 73-88. Recuperado de https://www.redalyc.org/articulo.oa?id=4137/413740745008

Rueda, S. (2014). P5 Modelos de ordenación del territorio más sostenibles. Boletín CF+ S, (32-33), 119-134. Recuperado de http://polired.upm.es/index.php/boletincfs/article/view/2340/2422

Universidad de Ibagué. (2020). I Congreso Internacional de Desarrollo Regional. Recuperado de https://extension.unibague.edu.co/i-congreso-internacional-de-desarrollo-regional

Villaveces-Niño, M. J., \& Sánchez, F. (2015). Tendencias históricas y regionales de la adjudicación de baldíos en Colombia. Recuperado de https://repository.urosario.edu.co/bitstream/handle/10336/10933/12538.pdf?sequence 